\title{
Comparative WGBS identifies genes that influence non-ripe phenotype in tomato epimutant Colourless non-ripening
}

Weiwei Chen ${ }^{1 \dagger}$, Zhiming $\mathrm{Yu}^{1 \dagger}$, Junhua Kong ${ }^{1 \#}$, Hui Wang ${ }^{1}$, Yichen $\mathrm{Li}^{2}$, Mei Zhao ${ }^{1}$, Xiaohong Wang ${ }^{1}$, Qianqian Zhen ${ }^{1}$, Nongnong Shi ${ }^{1}$, Pengcheng Zhang ${ }^{1}$, Silin Zhong ${ }^{2}$, Paul Hunter ${ }^{3}$, Mahmut Tör ${ }^{4}$, Yiguo Hong ${ }^{1,3,4^{*}}$

${ }^{1}$ Research Centre for Plant RNA Signaling, College of Life and Environmental Sciences, Hangzhou Normal University, Hangzhou 310036, China.

${ }^{2}$ State Key Laboratory of Agrobiotechnology, School of Life Sciences, The Chinese University of Hong Kong, Hong Kong, China.

${ }^{3}$ Warwick-Hangzhou Joint RNA Signaling Laboratory, School of Life Sciences, University of Warwick, Warwick CV4 7AL, UK.

${ }^{4}$ Worcester-Hangzhou Joint Molecular Plant Health Laboratory, Institute of Science and the Environment, University of Worcester, WR2 6AJ, UK

Short title: Epi-genotype to epi-phenotype in Cnr fruits

${ }^{\dagger}$ Contributed equally to this work.

*Corresponding author (email: yiguo.hong@hznu.edu.cn, yiguo.hong@warwick.ac.uk)

\#Current Address: UMR EGFV, Bordeaux Sciences Agro, INRA, Université de Bordeaux, 210 Chemin de Leysotte, CS 50008, 33882 Villenave d’Ornon, France. 
Whole-genome bisulfite sequencing (WGBS) allows single-base resolution and genome-wide profiling of DNA methylation in plants and animals. This technology provides a powerful tool to identify genes that are potentially controlled by dynamic changes of DNA methylation and demethylation. However naturally occurring epimutants are rare and genes under epigenetic regulation as well as their biological relevances are often difficult to define. In tomato, fruit development and ripening are a complex process that involves epigenetic control. We have taken the advantage of the tomato epimutant Colourless non-ripening ( $\mathrm{Cnr}$ ) and performed comparative mining of the WGBS datasets for the Cnr and SICMT3-silenced Cnr fruits. We compared DNA methylation profiles for the promoter sequences of approximately 5,000-bp immediately upstream of the coding region of a list of 20 genes. Differentially methylated regions were found for some of these genes. Virus-induced gene silencing (VIGS) of differentially methylated gene SIDET1 or SIPDS resulted in unusual brown pigmentation in Cnr fruits. These results suggest that comparative WGBS coupled with VIGS can be used to identify genes that may contribute to the colourless unripe phenotype of fruit in the Cnr epimutant.

\section{Tomato Cnr, SICMT3, SIDET1, SIPDS, DNA Methylation, WGBS, VIGS}

\section{INTRODUCTION}

Tomato (Solanum lycopersicum) is a nutritional fruit and vegetable crop that is consumed worldwide. It also serves as a model species to investigate mechanisms involved in the modulation of fleshy fruit ripening (Klee and Giovannoni, 2011). From characterisation of various ripening mutants, it has become evident that fruit ripening is a complex process at physiological, genetic and molecular levels (Seymour et al., 2013). For instance, mapping the ripening inhibitor (rin) locus reveals a gene that encodes a MADS-box protein designated SIMADS-RIN (Vrebalov et al., 2002), and that SIMADS-RIN acts as a master transcription factor (TF) in regulation of many ripening-related genes in tomato (Martel et al., 2011; Zhou et al, 2012; Fujisawa et al., 2013). The class-I homeodomain leucine zipper protein SlHB-1 is another TF that regulates fruit ripening through its direct binding to the 1aminocyclopropane-1-carboxylate oxidase (ACO) gene promoter, resulting in a tight transcriptional control of ACO1 expression. The latter is an essential component in the 
genetic pathway for biosynthesis of the ripening hormone ethylene (Lin et al., 2008). Several other TFs including SIAP2a and SITAGL1 have also been showed to play important roles in fruit ripening, indicating existence of a genetic regulatory network associated with ripening in tomato. However, how these ripening TFs are regulated and which genes are targeted and regulated by these TFs remain largely to be elucidated in tomato (Karlova et al., 2014).

Another key tomato ripening gene, namely the SQUAMOSA Promoter Binding Protein (SBP)-box gene SISPL-CNR resides at the Colourless non-ripening ( $\mathrm{Cnr}$ ) locus (Manning et al., 2006; Kong et al., 2013). Cnr is a spontaneous pleiotropic epimutant in which SISPL-CNR expression is affected by DNA methylation in its promoter region and is also finely tuned by miR157 (Manning et al., 2006; Chen et al., 2015a). Cnr fruit cannot ripen and remains colourless. Its texture alters due to loss of cell-to-cell adhesion in fruit tissues (Eriksson et al., 2004). Intriguingly, enzymes that are required for RNA-directed DNA methylation (RdDM) and for methylation maintenance including DOMAINS REARRANGED METHYLTRANSFERASE 7 (SIDRM7), METHYLTRANSFERASE 1 (SIMET1) and CHROMOMETHYLASEs (SICMT2 and SICMT3), in particular SICMT3, are essential to maintain the $\mathrm{Cnr}$ epiallele and the colourless non-ripening epi-phenotype (Chen et al., 2015b). Consequently, repression of these methylation genes by virus-induced gene silencing (VIGS) can lead Cnr fruits to ripen (Chen et al., 2015b). These findings clearly demonstrate that an epigenetic mechanism is involved in Cnr fruit development and ripening. This view is further supported by the finding that dynamic changes in the tomato epigenomes occur at different stages of fruit development and ripening in wild-type tomato as well as in rin and Cnr mutants (Zhong et al., 2013; Chen et al., 2015b; Zhang et al., 2016). Moreover active DNA demethylation has also been found to play a vital role in modulation of tomato fruit ripening (Liu et al., 2015; Lang et al., 2017). These recent whole-genome studies have revealed that tomato development and fruit ripening are not only genetically but also epigenetically programmed, and can be influenced by many potential genes that may be affected by DNA methylation and demethylation (Gullasci et al., 2016; Giovannoni et al., 2017). However specific fruit ripening genes under epigenetic regulation remain to be identified and functionally characterized in tomato.

In this article, we report characterization of genes that are associated with tomato ripening through comparative mining of the whole-genome bisulfite sequencing (WGBS) datasets that we previously generated for the epiallele Cnr non-ripe fruits and the SICMT3silenced Cnr ripening fruits (Chen et al., 2015b). In particular we compared DNA methylation profiles for the promoter sequences of approximate 5,000-bp immediately 
upstream of the coding region of each gene. Differentially methylated regions (DMRs) were found for some of these genes and silencing of two of the differentially methylated genes by VIGS affected pigmentation in Cnr fruits. These results suggest that comparative WGBS coupled with VIGS can be used to identify genes that may contribute to colourless non-ripe epi-phenotype in Cnr fruits.

\section{RESULTS}

Cnr with an epigenome of hypermethylation is caused by a spontaneous epimutation that blocks the expression of SISPL-CNR at different stages of fruit development and ripening (Manning et al., 2006; Zhong et al., 2013; Figure 1A). We previously demonstrated that suppression of SICMT3 by VIGS caused Cnr fruits to ripen, likely due to a specific reduction of the DNA methylation level in the SISPL-CNR promoter coupled with an overall decrease of methylation in the Cnr epigenomes (Chen et al., 2015b). Repeating our VIGS experiments, we further established that SICMT3 VIGS is responsible for the ripening reversion in the treated Cnr fruits (Figure 1B). Using the latest tomato genome and epigenome databases, we also confirmed that silencing of SICMT3 reduced the DNA methylation level in the two differentially methylated regions (DRM1 and DRM2) within the SISPL-CNR promoter in the VIGS fruits compared to non-VIGS Cnr controls (Figure 1C). Although Cnr is a dominant epiallele and $S I S P L-C N R$ is primarily responsible for the phenotypic colourless non-ripening in $\mathrm{Cnr}$ fruits, we reason that other genes might also contribute to the development of such epi-phenotypes and those genes can be identified via comparative mining of the WGBS datasets generated from the $\mathrm{Cnr}$ non-ripe fruits and the SICMT3-silenced $\mathrm{Cnr}$ ripening fruits.

To test this, we compiled a list of 20 genes (Table 1) and these genes were chosen because they have been implicated to be directly or indirectly associated with tomato development and fruit ripening (Karlova et al., 2014). We examined changes of the DNA methylation patterns for each of these selected genes, particularly in the 5,000-bp promoter sequences prior to the gene coding region. One or more obvious DMRs were readily identified in 12 genes including SISPL-CNR, SINOR, SIMADS-RIN, SITAGL1 and SIPDS known to be linked with tomato fruit ripening (Table 1). These DMRs were hypermethylated in normal $\mathrm{Cnr}$ fruits compared to that in wild-type tomato Ailsa Craig (AC) and mutant rin fruits. However in the SICMT3-silenced Cnr fruits with ripening phenotypes (Figure 1B), DNA methylation levels in each of the corresponding DMRs were clearly reduced. For instance, one such DMR was found in the promoter region of the MADS-box gene 
SIFUL1/SITDR4 and three (DMR1, DMR2 and DMR3) in the promoter region of the GRAS family SIGRAS gene (Figure 2A and B). Expression levels of both SIFUL1/SITDR4 (Figure 2C) and SIGRAS (Figure 2D) revealed by RNAseq were constantly very low in Cnr fruits at different days post anthesis (DPA). However, in AC fruits only hypomethylation was observed in these DMRs (Figure 2A and B) and expression levels of the two genes ware relatively high at later stages of fruit development and ripening (Figure 2C and D). We interpret these results to mean that such ripening-associated genes are epigenetically regulated and that they may contribute to the colourless non-ripening epi-phenotypes in $C n r$ fruits.

However, not all of the ripening genes showed changes in DNA methylation in the SICMT3-silenced Cnr ripening fruits. Indeed, no obvious DMR could be located for genes such as SIAP2a, SIMADS1, SIFUL2, SISEP3 and SIFBP24-Like through scrutinising the Cnr and SICMT3-silenced Cnr WGBS datasets (Table 1). These analyses imply that although the five genes may play important roles in fruit ripening in wild-type tomato, in $C n r$ they are not necessarily under the RdDM-mediated epigenetic control and they are unlikely to contribute to the unripe epi-phenotypes. On the other hand, in the case of SITAG1, we observed that decrease and increase in DNA methylation concurrently occurred within the two DMRs in the SICMT3-silenced Cnr ripening fruits (Figure 3A). Interestingly, the level of the SITAG1 RNA transcripts was relatively abundant in both $\mathrm{Cnr}$ and AC, but higher in $\mathrm{Cnr}$ than AC at 7, 17 and 47-DPA; similar at 37 and 42-DPA; however at 27-DPA more expression of SITAG1 was observed in AC (Figure 3C). It seems that there is no clear correlation between SITAG1 expression, SITAG1 promoter methylation and fruit ripening in Cnr, SICMT3-silenced Cnr, rin and AC. A similar pattern of DNA methylation changes in DMRs was also observed for SIANT1 in the SICMT3-silenced Cnr fruits (Table 1). Taken together, our analyses suggest both SITAG1 and SIANT1 may not make significant contributions to the development of epiphenotypes in Cnr fruits.

Through mining the WGBS datasets, we also identified an MYB TF gene SIAN2 which is involved in regulating expression of genes required for anthocyanidin biosynthesis (Povero et al., 2010; Kiferle et al., 2015). Compared to AC and rin, the DMR in the SIAN2 gene promoter was almost completely free of DNA methylation in normal Cnr fruit. However in the SICMT3-silenced Cnr ripening fruits, we observed an escalated methylation level in this DMR (Figure 3B). Moreover the SIAN2 mRNA level was extremely low in AC, Cnr and 
all other tomato mutant fruits (Figure 3D). These findings may imply that a highly methylated DMR cannot be accessed by a ripening-attenuator(s) in AC, SICMT3-silencing $\mathrm{Cnr}$, or even rin fruit. However in normal $\mathrm{Cnr}$, such a ripening-attenuator may bind to a methylation-free "DMR" in the SIAN2 promoter to affect SIAN2 expression, subsequently contributing to the colourless non-ripening epi-phenotypes. In addition, it should be noted that the level of anthocyanidin in tomato fruits is low and the role of anthocyanidin as well as relevant genes including SIAN2 for anthocyanidin biosynthesis in ripening remains unclear in tomato (Mathews et al., 2003; Povero et al., 2010).

Our analyses reveal that the 20 targeted genes fall into four different groups with respect of their DMRs in response to suppression of SICMT3 expression in Cnr; and that in addition to SISPL-CNR, other Group-I genes are also likely to be associated with the Cnr epiphenotypes (Table 1). To test this prediction drawn from our comparative mining of the WGBS datasets, we used VIGS to silence SIDET1 and SIPDS, two Group-I genes in Cnr (Table 1; Figure 4; Figure 5). As observed among other Group-I genes, two DMRs (DMR1 and DMR2) were identified in the SIDET1 promoter and their methylation levels reduced in the SICMT3-silenced ripening Cnr fruits (Figure 4A and B), but only one such DMR was found in the SIPDS promoter (Figure 5A). SIDET1 expression level was found to be low at different ripening stages in AC, $\mathrm{Cnr}$, high pigment $(h p)$ or other ripening mutant tomato fruits (Figure 4C). It should be pointed out that the high pigmentation phenotype of the tomato $h p-2$ mutant is caused by a mutation in the SIDET1 gene (Mustilli et al., 1999). We then cloned two fragments, one covering the 5'-end and the other covering the 3'-end of the SIDET1 gene into a potato virus X-based VIGS vector (van Wezel et al., 2002) to produce PVX/SIDET1n and PVX/SIDET1c, respectively (Figure 4D). We generated RNA transcripts of PVX/SIDET1n, PVX/SIDET1c or PVX by in vitro transcription and injected viral RNAs into the carpopodium of Cnr fruits attached to the plant. In repeated VIGS experiments, fruits were injected at various stages of development on different trusses on the same plant and on different plants. We found that Cnr fruits that were injected with free PVX virus developed non-ripening phenotype, typical of normal Cnr fruits (Figure 4E). However on fruits injected with either PVX/SIDET1n (Figure 4F and G) or PVX/SIDET1c (Figure 4H and I), we observed the appearance of intensified brown pigmentation. Similarly, we also cloned a fragment of the SIPDS gene into PVX vector to generate PVX/SIPDS (Figure 5B). Sectors on $\mathrm{Cnr}$ fruits that were injected with PVX/SIPDS recombinant viral RNA transcripts developed 
brown pigmentation (Figure 5C-E). These data clearly demonstrate that silencing of either SIDET1 or SIPDS can disrupt development of the epi-phenotypes in Cnr fruits.

\section{DISCUSSION}

In plants, epigenetic RdDM takes place at cytosines in CG, CNG and CNN contexts (where $\mathrm{N}$ is $\mathrm{A}, \mathrm{T}$ or $\mathrm{C}$ ) and such methylation can be maintained through the combined enzymatic activity of DRMs, MET1 and CMTs. In Arabidopsis DRM2 catalyses de novo CG, CNG and CNN methylation; whilst CMT2 is also involved in establishing non-symmetrical CNN methylation. Maintenance of cytosine methylation at the CG, CNG or CNN site is mediated by MET1, CMT3 or DRM2, respectively. Such epigenetic modification can have a profound influence on plant growth and development as well as plant responses to stress and adaptation to changing environments (Gallusci et al., 2016; Giovannoni et al., 2017). However, naturally occurring epimutation is sporadic and genes under epigenetic regulation as well as their biological significances are often difficult to delineate (Manning et al., 2006). WGBS permits genome-wide DNA methylation profiling at single-base resolution and is a powerful technology to identify genes that may be controlled by dynamic changes of DNA methylation and demethylation (Zhong et al., 2003; Lang et al., 2017). We used a rare tomato epimutant Cnr and performed comparative mining of the WGBS datasets for the Cnr and SICMT3silenced Cnr fruits (Chen et al., 2015b), in order to identify genes, in addition to the dominant SISPL-CNR, that may be associated with the development of the Cnr epi-phenotypes.

Our analyses revealed that the 20 genes included in current study fall into four different groups with respect of their DMRs in response to suppression of SICMT3 expression in Cnr (Table 1). Group-I includes SISPL-CNR and 11 other ripening-associated genes, which showed a reduced methylation level in the DMRs in the SICMT3-silenced Cnr ripening fruits. These genes are likely to be epigenetically regulated and DNA methylation-mediated downregulation of expression of these genes may play a role in development of the non-ripening epi-phenotypes in Cnr. Using VIGS, we were able to functionally confirm that Group-I genes such as SIDET1 and SIPDS can affect the development of the Cnr phenotypes (Figure 4; Figure 5). However the impact of Group-II genes on Cnr fruit development and (non)ripening is difficult to predict due to fluctuating up- and down-changes in DNA methylation within their DMRs. On the other hand, the unexpected increase in methylation level for the Group-III gene DMR in the SlCMT3-silenced Cnr is intriguing, and may suggest a different mechanistic mode for this group of genes or genes with similar DMR methylation patterns, to 
epigenetically regulate epi-phenotypic development compared to Group-I genes in Cnr.

Finally Group-IV genes, even they are known to be involved in ripening in wild-type or other ripening-mutant tomatoes, would be unlikely to play a role in the development of the Cnr epiphenotypes (Table 1).

In summary, through mining WGBS datasets for the epiallele Cnr non-ripe fruits and the SlCMT3-silenced $\mathrm{Cnr}$ ripening fruits as well as gene functional analysis via the efficient virus-induced gene silencing system, we were able to characterize genes that are under RdDM-mediated epigenetic modulation and associated with development of the Cnr epiphenotypes.

\section{MATERIALS AND METHODS}

\section{Plant materials and growth}

Tomato (Solanum lycopersicum cultivar Ailsa Craig, AC) and the Cnr epimutant plants were grown in insect-free glasshouses at $25^{\circ} \mathrm{C}$ during daytime with supplementary lighting to give a 16 -h photoperiod, and at $20^{\circ} \mathrm{C}$ overnight (8-h).

\section{Constructs of VIGS vectors}

Non-translatable fragments corresponding to the 5'-end (463bp) or 3'-end (390bp) of SIDET1 were PCR-amplified using two sets of primers PP376/ClaI (5’attcgATCGATgtggaatgaagctgaccaaac-3') and PP377/EagI (5’gaaggaCGGCCGgactgacaactacaaggcaaggaa-3') or PP374/ClaI (5'attcatATCGATgagacaacccaatatcctgaaat 3') and PP375/EagI (5'taatccCGGCCGccatactaaccgtcttggcactct-3’) and cloned into the ClaI/EagI sites of the Potato virus X (PVX) vector (van Wezel et al., 2002) to generate PVX/SIDET1n and PVX/SIDET1c, respectively. Similarly, a fragment of $483 \mathrm{bp}$ of the SIPDS gene was PCR-amplified using a pair of primers PP383/ClaI (5'-gccaggATCGATgagccgctttgatttct-3') and PP383/EagI (5'tcgtaaCGGCCGtctgacttggccaccttttgactc-3') and cloned into the ClaI/EagI sites of the PVX vector to produce PVX/SIPDS. All constructs were verified by sequencing. PVX/SICMT3 was generated in our previous study (Chen et al., 2015b).

\section{Whole-genome bisulfite sequencing (WGBS) datasets and bioinformatics analysis}


WGBS datasets for Cnr, SICMT3-silenced Cnr as well as the wild-type tomato Ailsa Craig (AC) and the rin mutant were available from our previous studies (Zhong et al., 2013; Chen et al., 2015b; http://www.epigenome.cuhk.edu.hk/encode.html). Bioinformatics analysis was performed as previously described (Zhong et al., 2013). Specific gene identification numbers were obtained from the tomato genome database (https://solgenomics.net/search/locus). Transcript expression levels of each gene at different days post-anthesis were calculated from the RNA transcriptome databases (http://www.epigenome.cuhk.edu.hk/ZhongWeb/tomato/tomato_index.jsp).

\section{Virus-induced gene silencing (VIGS)}

PVX-based VIGS in Cnr fruits was performed as described (Chen et al., 2015b). Viral RNA transcripts were generated by in vitro transcription as described (Zhou et al., 2012). The carpopodium of $\mathrm{Cnr}$ fruits at 5-15 days post anthesis was needle-injected with recombinant viral RNAs for each of the PVX-based VIGS constructs. After injection Cnr fruits were routinely examined and photographed with a Nikon Coolpix995 digital camera (Chen et al., 2015b).

Compliance and ethics The authors declare that they have no conflict of interest.

Acknowledgements We are indebted to the two editors Yijun Qi and Jiawei Wang of Science China Life Sciences for inviting us to contribute a research article to this Special Issue. We thank Kenneth Manning for his initial involvement in this project and critical reading of this manuscript. We are grateful to David Baulcombe for his kind gift of the original PVX vector. This work was supported by grants from Ministry of Agriculture of the People's Republic of China (the National Transgenic Program of China 2016ZX08009001004 to Y.H.); National Natural Science Foundation of China (NSFC 31370180 to Y.H., 31601765 to W.C.); Hangzhou Normal University (Pandeng Program 201108 to Y.H.); the Hangzhou City Government (Innovative Program for Science Excellence 20131028 to Y.H.); Zhejiang Provincial Natural Science Foundation (LY14C010005 to N.S.), and the UK Biotechnology and Biological Sciences Research Council (BBS/E/H/00YH0271 to Y.H.).

Chen, W., Kong, J., Lai, T., Manning, K., Wu, C., Wang, Y., Qin, C., Li, B., Yu, Z., Zhang, X., He, M., Zhang, P., Gu, M., Yang, X., Mahammed, A., Li, C., Osman, T., Shi, N., Wang, H., Jackson, S., Liu, Y., Gallusci, P., and Hong, Y. (2015a). Tuning LeSPL-CNR expression by SlymiR157 affects tomato fruit ripening. Sci Rep 5, 7852. 
Chen, W., Kong, J., Qin, C., Yu, S., Tan, J., Chen, Y.R., Wu, C., Wang, H., Shi, Y., Li, C., Li, B., Zhang, P., Wang, Y., Lai, T., Yu, Z., Zhang, X., Shi, N., Wang, H., Osman, T., Liu, Y., Manning, K., Jackson, S., Rolin, D., Zhong, S., Seymour, G.B., Gallusci, P., and Hong, Y. (2015b). Requirement of CHROMOMETHYLASE3 for somatic inheritance of the spontaneous tomato epimutation Colourless non-ripening. Sci Rep 5, 9192.

Eriksson EM, Bovy A, Manning K, Harrison L, Andrews J, De Silva J, Tucker GA, Seymour GB. (2004). Effect of the Colorless non-ripening mutation on cell wall biochemistry and gene expression during tomato fruit development and ripening. Plant Physiol 136, 4184-4197.

Fujisawa, M., Nakano, T., Shima, Y., and Ito, Y. (2013). A large-scale identification of direct targets of the tomato MADS box transcription factor RIPENING INHIBITOR reveals the regulation of fruit ripening. Plant Cell 25, 371-386.

Giovannoni, J., Nguyen, C., Ampofo, B., Zhong, S., and Fei Z. (2017). The epigenome and transcriptional dynamics of fruit ripening. Annu Rev Plant Biol 68, 61-84.

Gallusci, P., Hodgman, C., Teyssier, E., and Seymour, G.B. (2016). DNA methylation and chromatin regulation during fleshy fruit development and ripening. Front Plant Sci 7, 807.

Karlova, R., Chapman, N., David, K., Angenent, G.C., Seymour, G.B., and de Maagd, R.A. (2014). Transcriptional control of fleshy fruit development and ripening. J Exp Bot 65, 4527-4541.

Kiferle, C., Fantini, E., Bassolino, L., Povero, G., Spelt, C., Buti, S., Giuliano, G., Quattrocchio, F., Koes, R., Perata, P., and Gonzali, S. (2015). Tomato R2R3-MYB proteins SlANT1 and SlAN2: Same protein activity, different roles. PLoS One 10, e0136365.

Klee HJ, Giovannoni JJ. (2011). Genetics and control of tomato fruit ripening and quality attributes. Annu Rev Genet 45, 41-59.

Kong, J., Chen, W., Shen, J., Qin, C., Lai, T., Zhang, P., Wang, Y., Wu, C., Yang, X., Hong, Y. (2013). Virus-induced gene complementation in tomato. Plant Signal Behav 8, e27142.

Lang, Z., Wang, Y., Tang, K., Tang, D., Datsenka, T., Cheng, J., Zhang, Y., Handa, A.K., and Zhu, J.K. (2017). Critical roles of DNA demethylation in the activation of ripening-induced genes and inhibition of ripening-repressed genes in tomato fruit. Proc Natl Acad Sci USA 114, E4511-E4519.

Lin, Z., Hong, Y., Yin, M., Li, C., Zhang, K., and Grierson, D. (2008). A tomato HD-Zip homeobox protein, LeHB-1, plays an important role in floral organogenesis and ripening. Plant J 55, 301-310.

Liu, R., How-Kit, A., Stammitti, L., Teyssier, E., Rolin, D., Mortain-Bertrand, A., Halle, S., Liu, M., Kong, J., Wu, C., Degraeve-Guibault, C., Chapman, N.H., Maucourt, M., Hodgman, T.C., Tost, J., Bouzayen, M., Hong, Y., Seymour, G.B., Giovannoni, J.J., and Gallusci, P. (2015). A DEMETERlike DNA demethylase governs tomato fruit ripening. Proc Natl Acad Sci USA 112, 10804-10809.

Martel, C., Vrebalov, J., Tafelmeyer, P., and Giovannoni, J.J. (2011). The tomato MADS-box transcription factor RIPENING INHIBITOR interacts with promoters involved in numerous ripening processes in a COLORLESS NONRIPENING-dependent manner. Plant Physiol 157, 1568-1579. 
Manning, K., Tör, M., Poole, M., Hong, Y., Thompson, A.J., King, G.J., Giovannoni, J.J., and Seymour, G.B. (2006). A naturally occurring epigenetic mutation in a gene encoding an SBP-box transcription factor inhibits tomato fruit ripening. Nat Genet 38, 948-952.

Mathews, H., Clendennen, S.K., Caldwell, C.G., Liu, X.L., Connors, K., Matheis, N., Schuster, D.K., Menasco, D.J., Wagoner, W., Lightner, J., and Wagner, D.R. (2003). Activation tagging in tomato identifies a transcriptional regulator of anthocyanin biosynthesis, modification, and transport. Plant Cell 15, 1689-1703.

Mustilli, A.C., Fenzi, F., Ciliento, R., Alfano, F., and Bowler, C. (1999). Phenotype of the tomato high pigment-2 mutant is caused by a mutation in the tomato homolog of DEETIOLATED1. Plant Cell 11, 145-157.

Povero, G., Gonzali, S., Bassolino, L, Mazzucato, A., and Perata, P. (2010). Transcriptional analysis in high-anthocyanin tomatoes reveals synergistic effect of Aft and atv genes. J Plant Physiol 168, 270-279.

Seymour, G.B., Østergaard, L., Chapman, N.H., Knapp, S., and Martin, C. (2013). Fruit development and ripening. Annu Rev Plant Biol 64, 219-241.

van Wezel, R., Dong, X., Liu, H., Tien, P., Stanley, J., and Hong, Y. (2002). Mutation of three cysteine residues in Tomato yellow leaf curl virus-China C2 protein causes dysfunction in pathogenesis and posttranscriptional gene-silencing suppression. Mol Plant Microbe Interact 15, 203-208.

Vrebalov J, Ruezinsky D, Padmanabhan V, White R, Medrano D, Drake R, Schuch W, Giovannoni J. (2002). A MADS-box gene necessary for fruit ripening at the tomato ripening-inhibitor (rin) locus. Science 296, 343-346.

Zhang B, Tieman DM, Jiao C, Xu Y, Chen K, Fe Z, Giovannoni JJ, Klee HJ. (2016). Chilling-induced tomato flavor loss is associated with altered volatile synthesis and transient changes in DNA methylation. Proc Natl Acad Sci USA 113, 12580-12585.

Zhong. S., Fei, Z., Chen, Y.R., Zheng, Y., Huang, M., Vrebalov, J., McQuinn, R., Gapper, N., Liu. B., Xiang, J., Shao, Y., and Giovannoni, J.J. (2013). Single-base resolution methylomes of tomato fruit development reveal epigenome modifications associated with ripening. Nat Biotechnol 31, 154-159.

Zhou, T., Zhang, H., Lai, T., Qin, C., Shi, N., Wang, H., Jin, M., Zhong, S., Fan, Z., Liu, Y., Wu, Z., Jackson, S., Giovannoni, J.J., Rolin, D., Gallusci, P., and Hong, Y. (2012). Virus-induced gene complementation reveals a transcription factor network in modulation of tomato fruit ripening. Sci Rep 2, 836. 


\section{Figure legends}

Figure 1 Differential DNA methylation in the SISPL-CNR promoter affects fruit ripening in the tomato epimutant Cnr. A, Expression level of SISPL-CNR during fruit ripening in wildtype tomato Ailsa Craig (AC), epimutant Cnr and mutants rin, hp and Nor. RPKM (Reads Per Kilobase of transcript per Million mapped reads) represents the relative expression level of SISPL-CNR transcript in RNAseq that is proportional to the number of cDNA fragments that originate from it. B, Ripening reversion in SICMT3-silenced Cnr fruits. Virus-induced SICMT3 gene silencing occurred in Cnr fruits that were injected with PVX/SICMT3 and ripened. Fruits were photographed at 54-days post anthesis (DPA). C, Reduction of DNA methylation in the specific promoter regions DMR1 and DMR2 of the SISPL-CNR gene in SICMT3-silenced Cnr. DMR refers to differentially methylated region. WGBS datasets for AC, rin, Cnr and SICMT3-silenced Cnr fruits at 42-DPA were used for comparative bioinformatics analysis. Gene ID for SISPL-CNR and its coordinate on tomato chromosome 2 are indicated.

Figure 2 Differential DNA methylation profiles of Group-I genes. A, Reduction of DNA methylation in the specific promoter region DMR of the SIFUL1/SITDR4 gene in SICMT3silenced Cnr. B, Decrease of DNA methylation in the specific promoter regions DMR1, DMR2 and DMR3 of the SIGRAS gene in SICMT3-silenced Cnr. DMR refers to differentially methylated region. WGBS datasets for AC, rin, Cnr and SICMT3-silenced Cnr fruits at 42 days post anthesis (DPA) were used for comparative bioinformatics analysis. Gene IDs and their coordinates on tomato chromosomes are indicated. C and D, Expression level of SIFUL1/SITDR4 (C) and SIGRAS (D) during fruit ripening in wild-type tomato Ailsa Craig (AC), epimutant Cnr and mutants rin, $h p$ and Nor. RPKM (Reads Per Kilobase of transcript per Million mapped reads) represents the relative expression level of mRNA transcript in RNAseq that is proportional to the number of cDNA fragments that originate from it.

Figure 3 Differential DNA methylation profiles of Group-II and Group III genes. A, DNA methylation dynamics in the specific promoter regions DMR1 and DMR2 of the SITAG1 gene in SICMT3-silenced Cnr. B, Increase of DNA methylation in the specific promoter region DMR of SIAN2 gene in SICMT3-silenced Cnr. DMR refers to differentially methylated region. WGBS datasets for AC, rin, Cnr and SICMT3-silenced Cnr fruits at 42 days post anthesis (DPA) were used for comparative bioinformatics analysis. Gene IDs and 
their coordinates on tomato chromosomes are indicated. C and D, Expression level of SITAG1 (C) and SIAN2 (D) during fruit ripening in wild-type tomato Ailsa Craig (AC), epimutant Cnr and mutants rin, $h p$ and Nor. RPKM (Reads Per Kilobase of transcript per Million mapped reads) represents the relative expression level of mRNA transcript in RNAseq that is proportional to the number of cDNA fragments that originate from it.

Figure 4 VIGS of Group-I gene SIDET1 affects pigmentation in Cnr fruits. A and B, Reduction of DNA methylation in the specific promoter regions DMR1 and DMR2 of the SIDET1 gene in SICMT3-silenced Cnr. DNA methylation profiles are shown for the entire gene (A) and for an enlarged section to show the reduced methylation in DMR1 and DMR2 (B). DMR refers to differentially methylated region. WGBS datasets for AC, rin, Cnr and SICMT3-silenced Cnr fruits at 42 days post anthesis (DPA) were used for comparative bioinformatics analysis. Gene IDs and their coordinates on tomato chromosomes are indicated. C, SIDET1 expression level at different stages of fruit ripening in AC, epimutant Cnr and mutants rin, $h p$ and Nor. RPKM (Reads Per Kilobase of transcript per Million mapped reads) represents the relative expression level of mRNA transcript in RNAseq that is proportional to the number of cDNA fragments that originate from it. D, VIGS constructs PVX/SIDET1n and PVX/SIDET1c. The PVX genome organization (RDRP: RNA-dependent RNA polymerase; the triple-gene block encodes $25 \mathrm{~K}, 12 \mathrm{~K}$ and $8 \mathrm{~K}$ movement proteins; CP: coat protein) and the two restriction enzymes ClaI and EagI are indicated. E-I, Phenotypes in control and SIDET1-silenced Cnr fruits. Cnr fruits were injected with the control PVX (E), PVX/SIDET1n (F and G), of PVX/SIDET1c (H and I), and photographed at 50-DPA.

Figure 5 VIGS of SIPDS affects pigmentation in Cnr fruits. A, Reduction of DNA methylation in differentially methylated region (DRM) of the SIPDS gene promoter in SICMT3-silenced Cnr. WGBS datasets for AC, rin, Cnr and SICMT3-silenced Cnr fruits at 42 days post anthesis (DPA) were used for comparative bioinformatics analysis. Gene IDs and their coordinates on tomato chromosomes are indicated. B, VIGS construct PVX/SIPDS. The PVX genome organization (RDRP: RNA-dependent RNA polymerase; the triple-gene block encodes $25 \mathrm{~K}, 12 \mathrm{~K}$ and $8 \mathrm{~K}$ movement proteins; CP: coat protein) and the two restriction enzymes ClaI and EagI are indicated. C-D, Phenotypes in SIPDS-silenced Cnr fruits. Fruit photograph was taken at 50-DPA. 